Gut, 1979, 20, 279-284

\title{
Endoscopic retrograde brush cytology in patients with primary and secondary malignancies of the pancreas
}

\author{
M. OSNES, A. SERCK-HANSSEN, O. KRISTENSEN, T. SWENSEN, S. AUNE, \\ AND J. MYREN
}

From the Departments of Medicine, Pathology, Radiology and Surgery, Ullevål Hospital, Oslo 1, Norway

SUMMARY Endoscopic retrograde brush cytology (ERBC) was performed in 29 patients with primary pancreatic malignancies verified later by histology. Results were positive in 21 patients, suspicious in five, and negative in three. ERBC performed in seven patients with uncertain findings at endoscopic retrograde cholangiopancreatography (ERCP) was positive for malignancy in six and suspicious in one. Endoscopic aspiration cytology (EAC) performed in 10 patients with primary tumours was positive for malignancy in three, suspicious in two, and negative in five cases. ERBC and/or EAC performed in five cases with secondary tumours were positive for malignancy in one and negative in four. It is concluded that ERBC gives a high rate of positive or suspicious $(90 \%)$ diagnosis of malignancy in patients with primary pancreatic lesions. In secondary tumours cytology seems to be negative in most cases. The study further shows that many of the malignant tumours found by ERP are secondary lesions. Cytology is often able to detect malignancy when ERP has proved inconclusive.

Pancreatic cytology may be obtained by duodenal aspiration (Asnaes and Johansen, 1970; Olsen, 1971), or by endoscopic cannulation of the main pancreatic duct (Endo et al., 1974; Hatfield et al., 1976) after exogenous pancreatic stimulation. In a previous publication (Osnes et al., 1975) we reported on our preliminary results using a special brush device which allows direct brush cytology of the pancreatic duct during endoscopic retrograde cholangiopancreatography (ERCP).

The purpose of the present publication was to present the results of endoscopic retrograde brush cytology (ERBC) and endoscopic retrograde aspiration cytology (EAC) in patients with histologically verified malignancies of the pancreas.

\section{Methods}

In a consecutive series of 69 patients (31 men, 38 women) aged 42-87 years (mean 68 years) with later histologically verified malignancies of the pancreas, ERBC was performed in 32 patients and EAC in 12. In three of these patients both examinations were performed. In 28 cases cytology was not obtained

Received for publication 25 October 1978 because of obvious changes due to pancreatic carcinoma $(n=12)$ beyond the reach of the brush device, technical difficulties $(n=11)$, or lack of cytological service or equipment to perform cytology $(n=5)$.

Ductal abnormalities were demonstrated by ERCP in all patients (Fig. 1), and were strongly suggestive of malignancy in 58 cases and uncertain in 11 patients. Specimens for histological examination were obtained during surgery in 45 cases and at necropsy in 24 cases. In 12 patients the malignancy were found to be secondary (Fig. 2, Table 1). The ductal abnormality was demonstrated in the head of the pancreas in 45 cases, in the body in 15 cases, and in the tail in nine cases. Islet cell tumours were found in two out of 57 patients with primary pancreatic lesions; the others were adenocarcinoma.

For ERBC a polyvinyl tube with side-viewing openings at the side were constructed as described in a previous paper (Osnes et al., 1975). A modification of this brush device (Fig. 3) with three instead of five side-viewing openings seems to be more suitable for retrograde brushing. For ERBC in patients with a narrow papillary opening a thinner brush, measuring $1.4 \mathrm{~mm}$ in diameter, has been constructed.

After the ductal abnormalities had been localised 


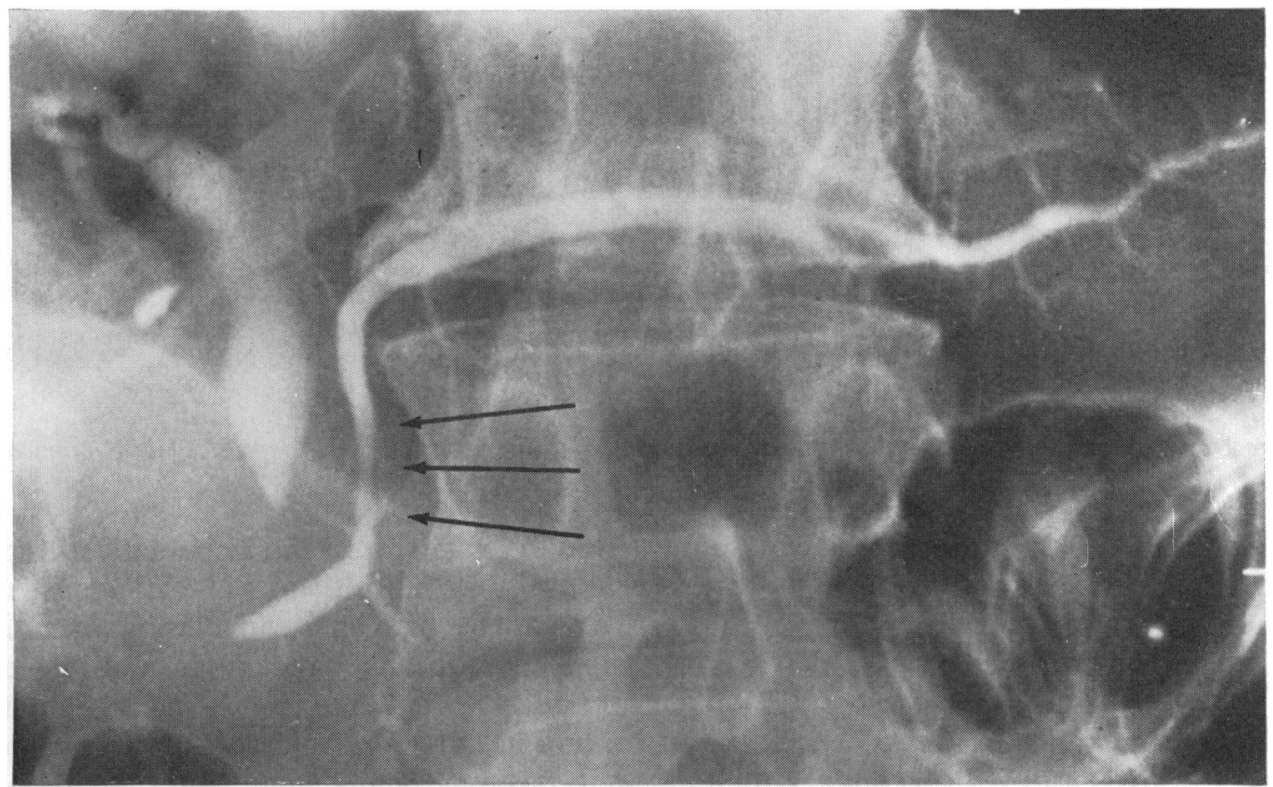

Fig. 1 Radiograph showing a stenotic pancreatic duct due to a primary adenocarcinoma of the pancreas.

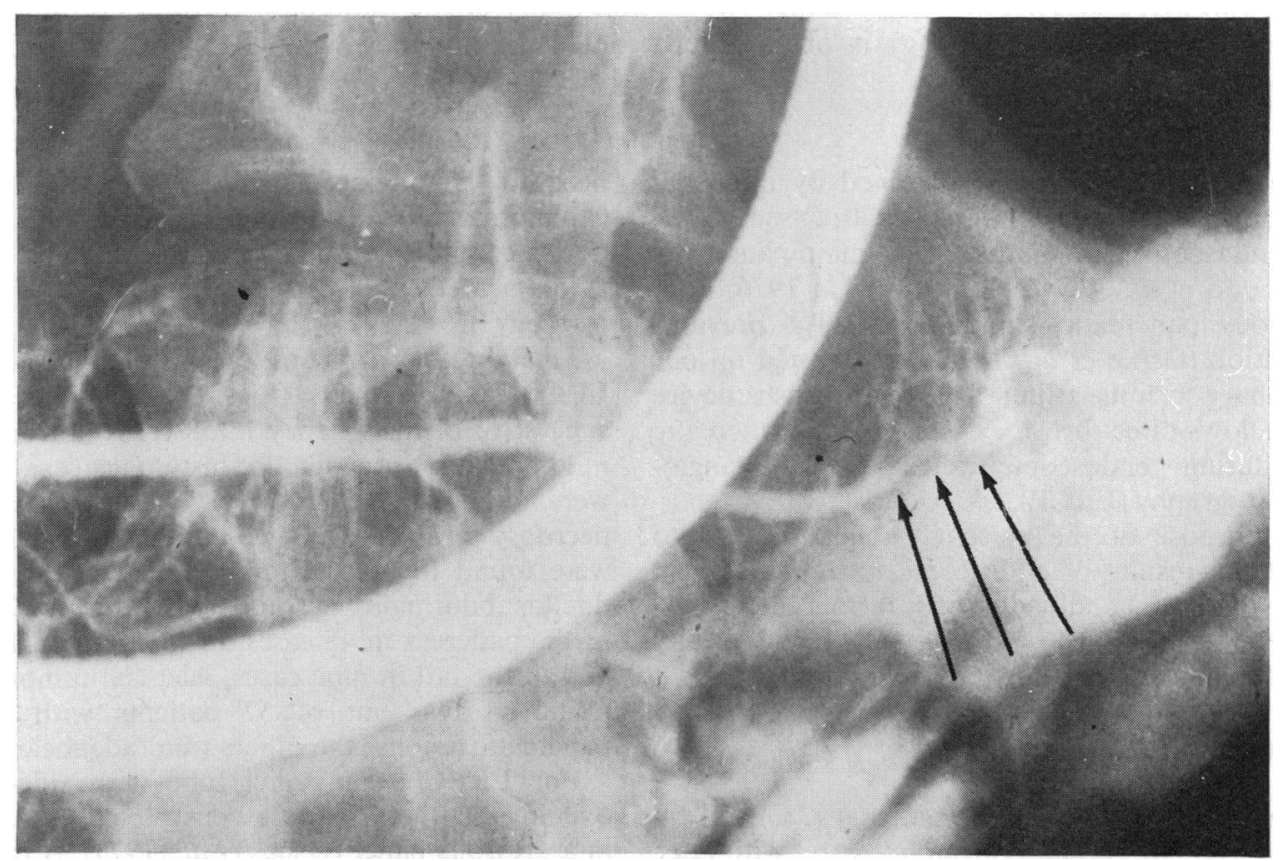

Fig. 2 Radiograph showing a stenotic pancreatic duct due to metastases from a bronchus carcinoma.

by ERCP the brush catheter was passed through the papilla of Vater (Fig. 4) and pushed as near as possible or into the ductal lesion (Fig. 5). When the brush had been withdrawn inside the duodenoscope this was immediately removed, the brush pushed out, and four to six smears made by rubbing the brush directly on glass slides. Smears were fixed before drying by a spray fixative and later stained 
Table 1 Primary localisation of secondary tumours found by endoscopic retrograde pancreatography (ERCP) $(N=12)$

\begin{tabular}{lllllll}
\hline Colon & Bronchus & Prostate & Stomach Liver & Testis & $\begin{array}{l}\text { Retroperi- } \\
\text { toneum }\end{array}$ \\
\hline 3 & 3 & 2 & 1 & 1 & 1 & 1 \\
\hline
\end{tabular}

Table 2 Results of endoscopic retrograde brush cytology in patients with primary and secondary malignancies of pancreas



according to Papanicolaou.

For EAC the patients were examined some days after endoscopic retrograde ductography. After exogenous pancreatic stimulation, juice was collected into test tubes containing $10 \%$ formalin. When it had been centrifuged two to four smears were made, fixed before drying, and stained as described above.

The cytological findings were classified according to Papanicolaou as negative for malignancy (I-II) (Fig. 6), suspicious (III), and positive for malignancy (IV-V) (Fig. 7).

All patients stayed in the hospital for at least twodays for clinical observation.

\section{Results}

RESULTS OF CYTOLOGICAL EXAMINATION IN RELATION TO HISTOLOGICAL FINDINGS ERBC was positive for malignancy in 21 patients

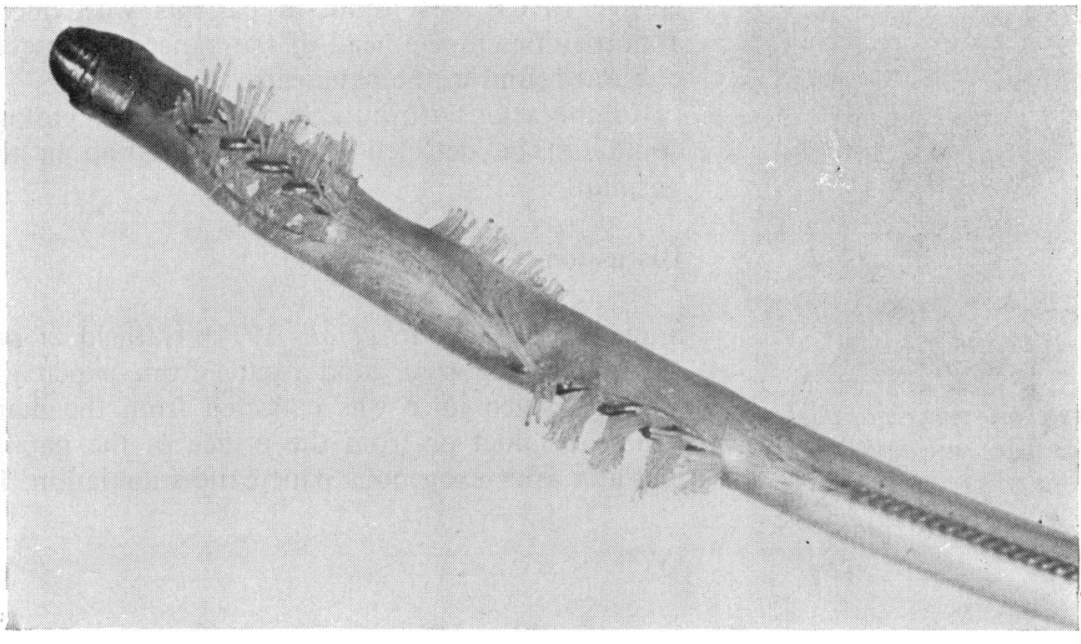

Fig. 3 The device used for endoscopic retrograde brush cytology (ERBC). Through a polyvinyl tube with side-viewing openings a soft brush is introduced. The distal end is plugged with a metal tip.
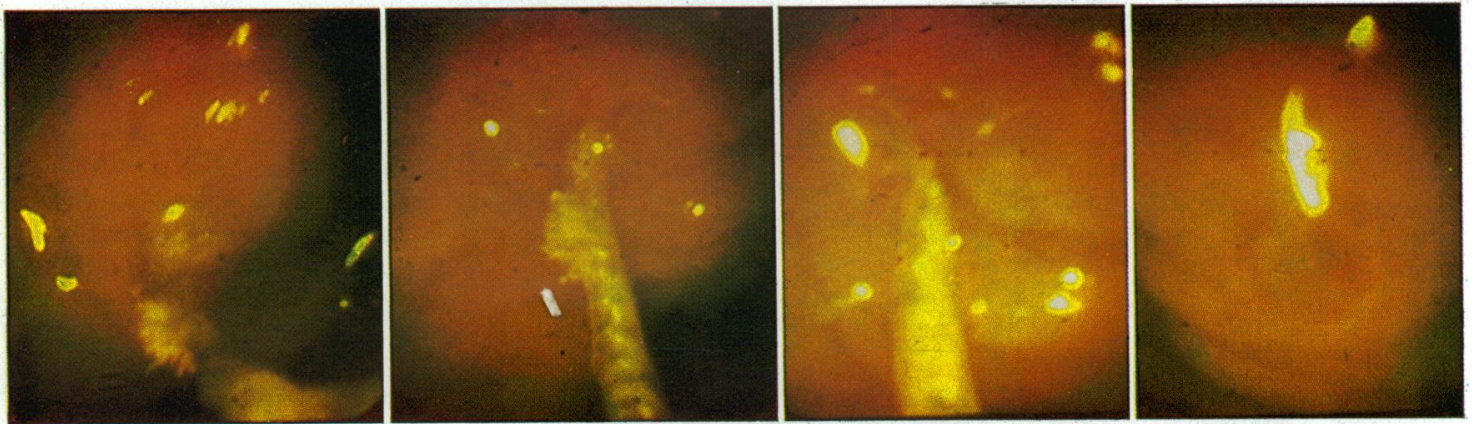

Fig. 4 Endoscopic picture showing endoscopic retrograde brush cytology (ERBC). The brush device is passed through the papilla of Vater under direct vision and fluoroscopic control. The right picture shows the papilla after the retrograde brushing. (Olympus instrument (JF B2).) 


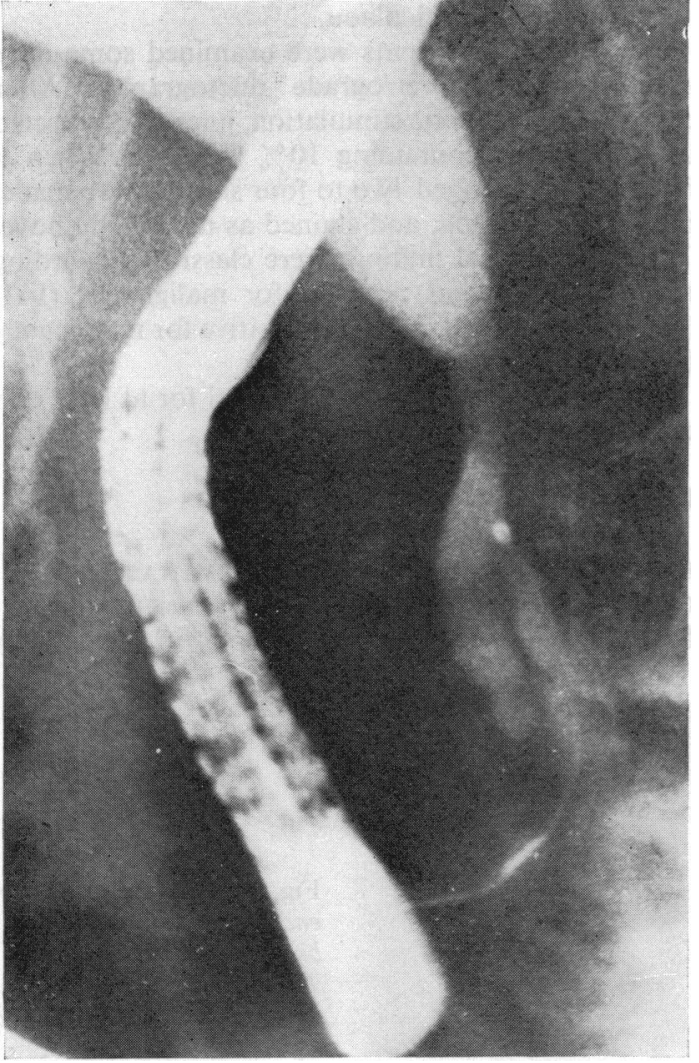

Fig. 5 Radiograph showing a stenotic pancreatic duct due to carcinoma of the pancreas. The brush device is pushed into the stenotic area of the pancreatic duct.
$(73 \%)$, suspicious in five $(17 \%)$, and negative in three $(10 \%)$ out of 29 patients with primary pancreatic lesions. In three patients with secondary tumours ERBC was positive in one and negative in two. EAC was positive for malignancy in three, suspicious in two, and negative in five patients with primary pancreatic lesions, whereas in two patients with secondary pancreatic tumours EAC was negative.

RESULTS OF CYTOLOGY IN RELATION TO FINDINGS BY ERCP

In patients with ductal abnormalities suggesting malignancies ERBC was positive in 15 out of 22 cases. In cases with uncertain findings at ERCP, ERBC was positive in six and suspicious in one out of seven patients on whom this method was used. EAC was positive in one and negative in two cases with uncertain findings at ERCP. Five out of six positive results by ERBC in patients with inconclusive ERCP was found in patients with ductal abnormalities in the head of the pancreas, whereas one was found in the pancreatic corpus.

Complications attributable to pancreatic cytology could not be detected during observation in the hospital.

\section{Discussion}

Some authors (Endo et al., 1974; Hatfield et al., 1976) have reported good results from pancreatic cytology when juice was collected from the main pancreatic duct or from the orifice of the papilla of Vater after exogenous pancreatic stimulation. In

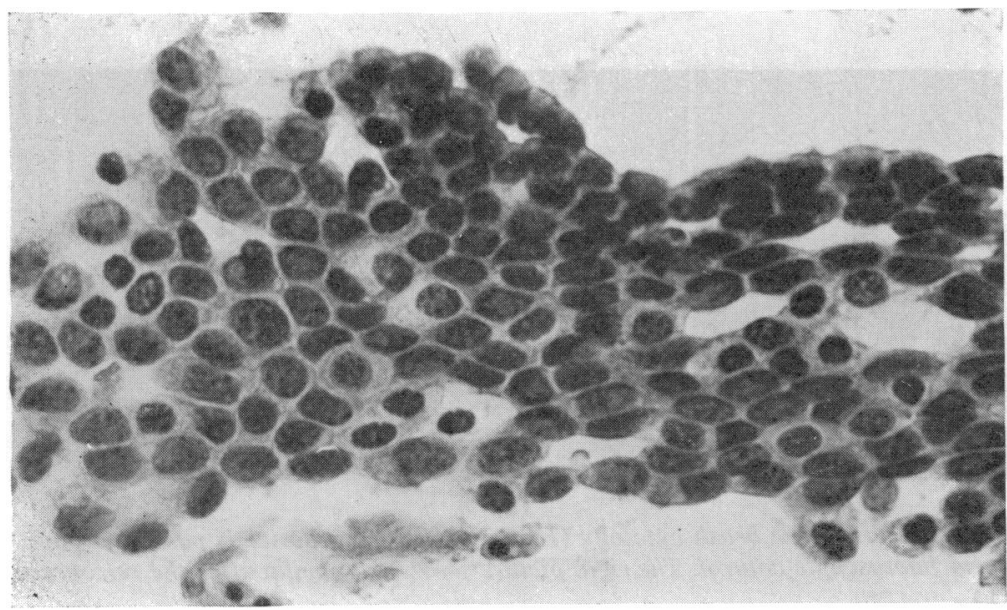

Fig. 6 Sheet of normal cells obtained from the pancreatic duct. Note regular size and appearance of cells with well marked cellular borders. Papanicolaou, $\times 600$. 


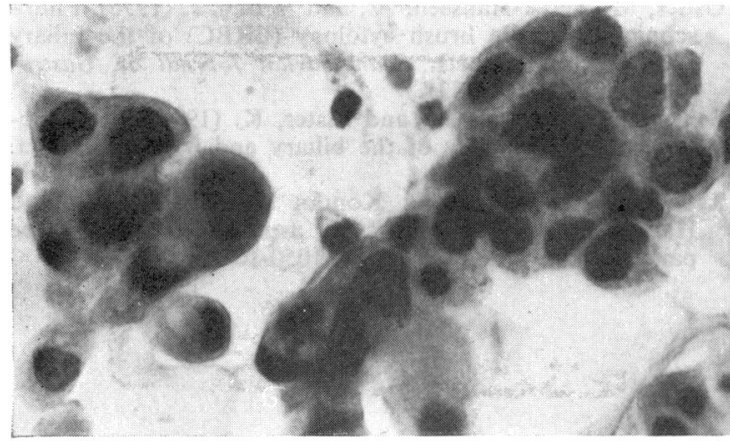

(a)



(b)

Fig 7. (a) Groups of malignant cells from the pancreatic duct in patient with moderately well-differentiated adenocarcinoma. Note hyperchromatic nuclei of varying size and shape. Papanicolaou, $\times 600$.

(b) Group of malignant cells from the pancreatic duct in patient with poorly differentiated adenocarcinoma. Great variation in amount of cytoplasm. Nuclei are vesicular with coarse chromatin and some have prominent nucleoli.

Papanicolaou, $\times 600$.

other studies (Cotton, 1977) the results of pancreatic cytology obtained by aspiration were rather poor in patients with pancreatic malignancies. In our series of patients with histologically verified primary pancreatic carcinoma, positive or suspicious smears were obtained in a high number using the brushing technique. On the other hand, the results of aspiration cytology were not convincing, but the number of patients examined by this technique is too small for firm conclusions. A comparison of the different techniques is not possible as we have examined only a few patients with both methods. The use of the brush device is, however, limited, as in many cases it is not possible to introduce it through the papilla of Vater, or the ductal lesions are beyond the reach of our brush for anatomical reasons. A sleeved brush previously used for bronchial cytology is now commercially available, but details of its use in the pancreatic duct are yet not available.

Devices for intraductal biopsies have been constructed (Seifert et al., 1977) and intraductal pancreatic biopsies have been attempted, but the rate of correct diagnosis in patients with pancreatic malignancies seems to be very low. A recent paper (Tsuchiya et al., 1977) reported good results of endoscopic transgastric and transduodenal aspiration cytology in patients with pancreatic malignancies.

The material obtained by ERBC is more abundant and contains cells that are better preserved than those obtained with ductal aspiration cytology. On the other hand, ductal brushing will not yield malignant cells unless the malignancy involves the mucosal surface of the main pancreatic duct or common bile duct. The observation that cytology was negative in most cases with secondary tumours may support this, although the numbers are too small for firm conclusions.

In the present series of patients ERCP was inconclusive with regard to malignancy in 11 patients. Brushing of the ductal abnormalities gave a positive result in six out of seven patients in this group, whereas one smear was suspicious regarding malignancy. Pancreatic cytology may be of great value in such cases.

As the use of endoscopic retrograde brushing probably should be limited to patients with ductal abnormalities, we feel that different sampling techniques should be used or added in patients without such demonstrable lesions.

\section{Conclusions}

Endoscopic retrograde brushing of ductal changes gives a high rate of positive or suspicious diagnosis in patients with primary pancreatic carcinomas. The technique is useful alongside ERCP in certain circumstances, and often clarifies an ERCP that does not give conclusive evidence of malignancy. This study also shows that a high number of malignant tumours found by ERCP are secondary lesions.

This study was supported by N. A. Stangs Legat and Helga Sembs Fond. The authors are grateful to $\mathrm{K}$. Porsbøll for preparing the figures.

\section{References}

Asnaes, S., and Johansen, A. (1970). Duodenal exfoliative cytology. Acta Pathologica et Microbiologica Scandinavica, suppl. 212, 11-14.

Cotton, P. B. (1977). ERCP. Gut, 18, 316-341.

Endo, Y., Morii, T., Tamura, H., and Okuda, S. (1974). Cytodiagnosis of pancreatic malignant tumors by aspira- 
tion, under direct vision, using a duodenal fiberscope. Gastroenterology, 67, 944-951.

Hatfield, A. R. W., Smithies, A., Wilkins, R., and Levi, A. J. (1976). Assessment of endoscopic retrograde cholangiopancreatography (ERCP) and pure pancreatic juice cytology in patients with pancreatic disease. Gut, 17, 14-21.

Olsen, J.H.(1971). Duodenal exfoliative cytology. Diagnosis of cancer of duodenum, pancreas, and biliary tract by exfoliative cytology. Scandinavian Journal of Gastroenterology, 6, suppl. 9, 105-109.
Osnes, M., Serck-Hanssen, A., and Myren, J. (1975). Endoscopic retrograde brush cytology (ERBC) of the biliary and pancreatic ducts. Scandinavian Journal of Gastroenterology, 10, 829-831.

Seifert, E., Urakami, Y., and Elster, K. (1977). Duodenoscopic guided biopsy of the biliary and pancreatic duct. Endoscopy, 9, 154-161.

Tsuchiya, R., Henmi, T., Kondo, N., Akashi, M., and Harada, N. (1977). Endoscopic aspiration biopsy of the pancreas. Gastroenterology, 73, 1050-1052. 